\title{
Non-inflammatory cerebral amyloid angiopathy as a cause of rapidly progressive dementia
}

\author{
A case study
}

\author{
Leonel Tadao Takada ${ }^{1,2}$, Paulo Camiz ${ }^{3}$, Lea T. Grinberg ${ }^{4,5}$, Claudia da Costa Leite ${ }^{6}$
}

\begin{abstract}
A 77 year-old men developed a subacute-onset, rapidly progressive cognitive decline. After 6 months of evolution, he scored 6 on the Mini-Mental State Examination and had left hemiparesis and hemineglect. The patient died 11 months after the onset of cognitive symptoms. Brain MRI showed microhemorrhages on gradientecho sequence and confluent areas of white matter hyperintensities on T2-weighted images. Brain biopsy revealed amyloid- $\beta$ peptide deposition in vessel walls, some of them surrounded by micro-bleeds. In this case report, we discuss the role of cerebral amyloid angiopathy (CAA) in cognitive decline, due to structural lesions associated with hemorrhages and infarcts, white matter lesions and co-morbidity of Alzheimer's disease, as well as the most recently described amyloid angiopathy-related inflammation.
\end{abstract}

Key words: dementia, cerebral amyloid angiopathy, vascular dementia.

\begin{abstract}
Angiopatia amilóide cerebral não inflamatória como causa de demência rapidamente progressiva: estudo de caso Resumo - Um homem de setenta e sete anos desenvolveu quadro de início subagudo de demência rapidamente progressiva. Após seis meses de evolução, sua pontuação do Mini exame do estado mental foi de 6 , e no exame neurológico apresentava heminegligência e hemiparesia esquerda. O paciente faleceu 11 meses após início dos sintomas. RM do encéfalo mostrou microhemorragias em sequência gradiente-echo e áreas confluentes de hiperintensidade de substância branca nas imagens pesadas em T2. Na biópsia cerebral observou-se deposição de peptídeo $\beta$-amilóide em vasos sanguíneos, alguns rodeados por microhemorragias. Neste relato de caso, discutimos o papel da angiopatia amiloide cerebral no declínio cognitivo, devido a lesões estruturais associadas a hemorragias e infartos, lesões de substância branca e comorbidade com doença de Alzheimer, assim como a mais recentemente descrita inflamação relacionada a angiopatia amiloide.

Palavras-chave: demência, angiopatia amiloide cerebral, demência vascular.
\end{abstract}

Rapidly progressive dementia (RPD), although more typically associated with prion diseases, can be caused by various conditions. ${ }^{1}$ These include neurodegenerative, autoimmune, toxic/metabolic and vascular etiologies, which must therefore be sought whenever investigating an RPD case. Here we present a case in which cerebral amyloid angiopathy (CAA) was found in a patient with RPD.

\section{Case report}

A seventy-seven year-old, highly educated (23 years of schooling) man was admitted to the geriatrics ward of our hospital for a six-month cognitive decline investigation. In January, 2008, his wife noticed a subacute difficulty in signing checks and reading. After about a month, he started to neglect his own medications. Three months later, he started presenting memory deficits, with rapid progression (within a month he could not remember whether he had had lunch). By that point, he was also having delusions (such as saying his late father was home) and aggressive behavior. By May 2008, he developed urinary incontinence

${ }^{1}$ Cognitive and Behavioral Neurology Unit, Hospital das Clínicas da Faculdade de Medicina da Universidade de São Paulo, São Paulo SP, Brazil. ${ }^{2}$ Centro de Referência em Distúrbios Cognitivos (CEREDIC), Hospital das Clínicas da Faculdade de Medicina da Universidade de São Paulo, São Paulo SP, Brazil. ${ }^{3}$ Geriatrics Department, Hospital das Clínicas da Faculdade de Medicina da Universidade de São Paulo, São Paulo SP, Brazil. ${ }^{4}$ Memory and Aging Clinics, Department of Neurology, University of California, San Francisco. ${ }^{5}$ Pathology Department, Faculdade de Medicina da Universidade de São Paulo, São Paulo SP, Brazil. ' Radiology Department, Hospital das Clínicas da Faculdade de Medicina da Universidade de São Paulo, São Paulo SP, Brazil.

Leonel Tadao Takada - Av. Paulista, 807 / cjto2515 - 01311-915 São Paulo SP - Brazil. E-mail: lttakada@hotmail.comDisclosure:

The authors report no conflicts of interest.

Received August 20, 2009. Accepted in final form October 16, 2009. 
and later, gait difficulty (despite using right leg prosthesis for 4 years, his wife denied previous gait abnormalities). No hallucinations or seizures were reported. At the time of evaluation he was completely dependent for basic daily life activities.

He had suffered from diabetes mellitus and systemic arterial hypertension for thirty years. In his past medical history, he had also undergone a right hip replacement and right transtibial amputation (for which he used a prosthesis for ambulation). He had suffered a head trauma with loss of consciousness at the age of 12 . No previous history of stroke was reported.

No family history of dementia was reported.

Upon neurological examination, he had hemineglect and anosognosia. His language skills appeared preserved, though he tended to confabulate and was dysarthric. His mini-mental state examination ${ }^{2}$ score was 6 (out of 30 ), having scored two points on spatial orientation, two points on immediate recall, one on naming and one on repetition. Semantic verbal fluency (animals) was zero. Bilateral ideomotor apraxia and optic ataxia were also seen. He was able to stand only with bilateral support and had left hemiparesis with deep tendon reflex asymmetry.

Blood testing revealed iron deficiency anemia (hemoglobin $10.8 \mathrm{~g} / \mathrm{dL}$ and hematocrit $33.9 \%$ ) and fasting blood glucose level of $202 \mathrm{mg} / \mathrm{dL}$ with a glycosylated hemoglobin level of 9.6\%. Anti-peroxidase and anti-tireoglobulin antibodies were below detection levels. Other biochemical tests, as well as vitamin B12 and folic acid levels, were normal. HIV and hepatitis B and C serology were negative. Erythrocyte sedimentation rate (ESR) was normal. Anti-neutrophil cytoplasmic antibody (ANCA), glutamic acid decarboxylase (GAD) antibodies, rheumatoid factor, anti-SS antibodies, serum protein electrophoresis, were all normal (or negative).
Anti-nucleus antibodies (ANA) were detected in 1:80 titers.

Spinal fluid analysis showed normal cellularity and increased protein levels $(60 \mathrm{mg} / \mathrm{dL})$, with slightly high gammaglobulin levels (14.9\%). Opening pressure was normal $\left(9 \mathrm{cmH}_{2} \mathrm{O}\right)$. Treponema pallidum immunology was negative (both in serum and spinal fluid).

Electroencephalogram showed diffuse disorganization of brain electrical activity and left temporal acute wave paroxysms. Gastrointestinal endoscopy revealed an active gastric ulcer, which was treated with proton pump inhibitor. Thoracic and abdominal computed tomography revealed no abnormal masses.

Neuroimaging findings are described below. During investigation, the spinal tap test was performed twice, but no cognitive or gait improvement was observed. Due to the rapidly progressive character of evolution, a right frontal cerebral biopsy was performed (also described below).

\section{Neuroimaging findings}

A MRI was performed on a 1.5 Tesla MR unit. The MRI protocol included: Axial diffusion-weighted image $(\mathrm{TR}=8000 \mathrm{~ms}, \mathrm{TE}=97 \mathrm{~ms}, \mathrm{~b}=0$ and $\mathrm{b}=1000$, and slice thickness $=5 \mathrm{~mm})$, axial T2* $(\mathrm{TR}=450 \mathrm{~ms}, \mathrm{TE}=15 \mathrm{~ms}$, flip angle $20^{\circ}$ and slice thickness $=5 \mathrm{~mm}$ ), axial unenhanced T1-weighted image ( $\mathrm{TR}=400 \mathrm{~ms}, \mathrm{TE}=11 \mathrm{~ms}$, and slice thickness $=5 \mathrm{~mm}$ ), axial FLAIR (fluid attenuated inversion recovery) image ( $\mathrm{TR}=11002 \mathrm{~ms}, \mathrm{TE}=103 \mathrm{~ms}, \mathrm{TI}=2400$ $\mathrm{ms}$, and slice thickness $=5 \mathrm{~mm}$ ), coronal T2-weighted fast spin echo $\mathrm{TR}=4730 \mathrm{~ms}, \mathrm{TE}=99 \mathrm{~ms}$, and slice thickness $=5$ $\mathrm{mm})$, and axial enhanced T1-weighted image ( $\mathrm{TR}=400$ $\mathrm{ms}, \mathrm{TE}=11 \mathrm{~ms}$, and slice thickness $=5 \mathrm{~mm})$ and axial volumetric spoiled gradient recalled echo (SPGR) $(\mathrm{TR}=6.6 \mathrm{~ms}$, $\mathrm{TR}=2.0$, slice thickness $=1.8 \mathrm{~mm}$, and interspace $=0.8 \mathrm{~mm}$ ) after gadolinium administration.

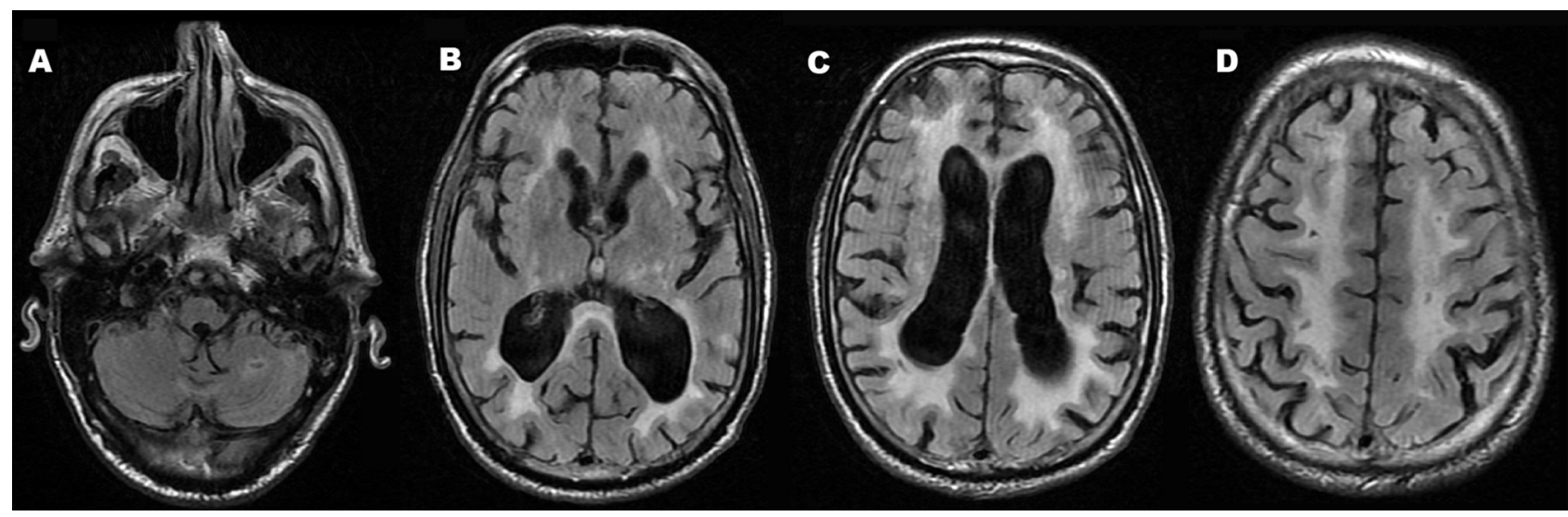

Figure 1. Axial FLAIR images. Note a hyperintense lesion with a hypointense core in the left cerebellar hemisphere [A]. There is ventricular dilatation and sulcal enlargement with multiple hyperintense lesions in the basal ganglia, thalamus, internal and external capsules and in the periventricular white matter $[B-C]$. There are also lesions in the subcortical area [D]. 


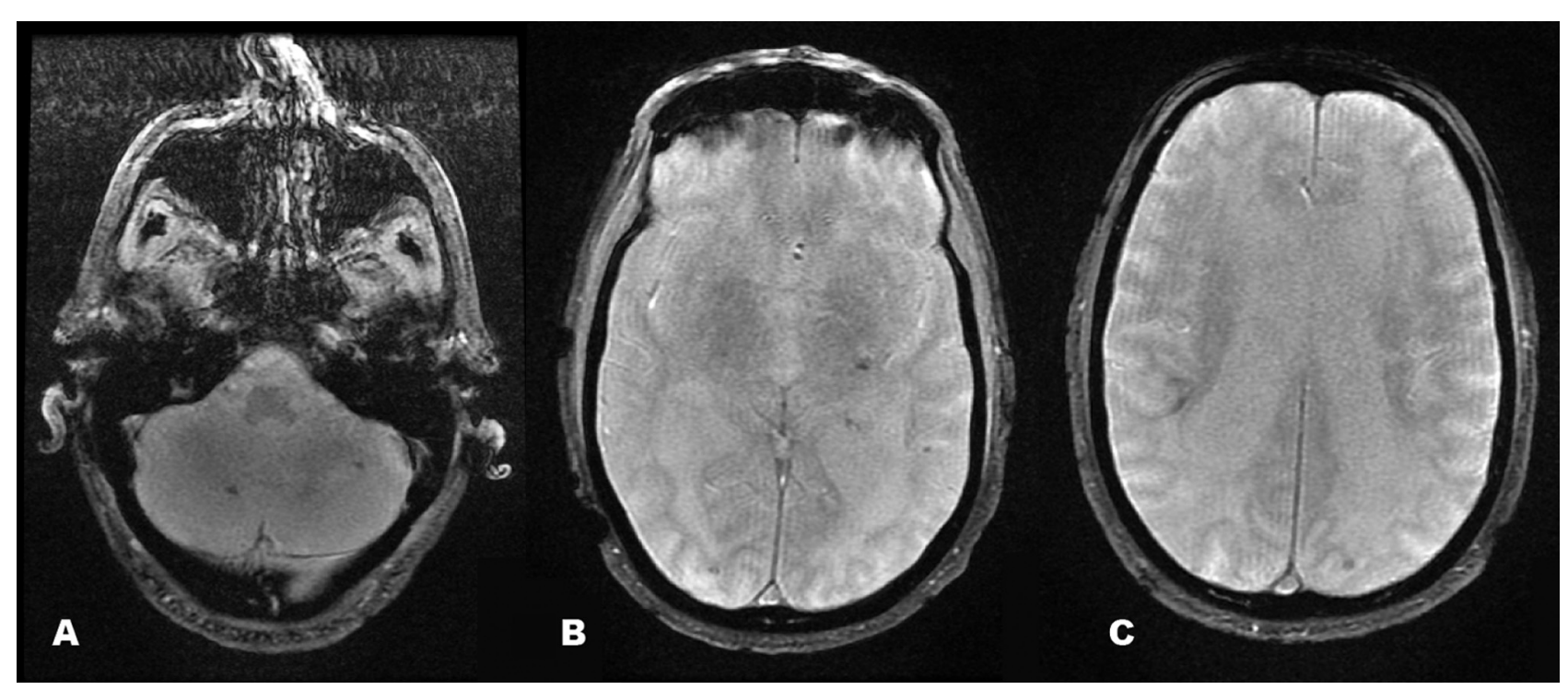

Figure 2. Axial T2* images showing multiple areas with marked hypointensity suggestive of hemossiderin content [A-C].

The MRI (Figure 1) showed ventricular dilatation and sulcal enlargement greater than the expected for the patient's age. The patient presented multiple hyperintense lesions in the cerebellum, pons, mesencephalon, basal ganglia, internal and external capsules, thalamus and periventricular, and subcortical white matter. These lesions may have represented microleukoangiopathy and/or gliosis. The $\mathrm{T}^{*}$ (Figure 2) images showed multiple marked hypointense foci suggestive of hemossiderin. The enhanced images showed no contrast enhancement. Computed tomography of the head demonstrated no calcified lesions.

\section{Neuropathological examination}

Three fragments of frontal cortex, the biggest measuring $0.7 \mathrm{~cm}$, were removed via stereotactic brain biopsy.

The small vessels of the gray matter were thickened by the deposition of amorphous material as detected using routine hematoxylin-eosin (HE) staining. Some of these vessels were surrounded by acute and semi-acute microbleedings (Figures $3 \mathrm{~A}$ and $3 \mathrm{~B}$ ).

Furthermore, the tissue was immunostained against: monoclonal mouse anti-Amyloid- $\beta$ 1:5000 (4G8, Covance, Emeryville, CA), monoclonal mouse anti-phospho-tau 1:1000 (PHF-1, gift of Peter Davies, New York, NY) and polyclonal rabbit anti-alpha-synuclein 1:10000 (EQV-1, gift of Kenji Ueda, Tokyo, Japan).

The anti-Amyloid- $\beta$ immunostaining revealed deposition of Amyloid- $\beta$ protein in the vessel walls of the cerebral gray matter corresponding to the amorphous material deposition observed on HE staining (Figure 3C). Scattered amyloid diffuse plaques were also seen in the neuropil.
Neuritic plaques, dystrophic neuritis and neurofibrillary tangles (NFT) and Lewy bodies were not seen in tissue.

The patient was diagnosed as having cerebral amyloid angiopathy with micro-bleedings. Due to the scarcity of available tissue, it was not possible to rule out Alzheimer's disease, despite the fact that neuritic plaques and neurofibrillary tangles were not found.

\section{Evolution}

After his discharge from hospital, the patient could no longer walk (even with aid), and developed pressure ulcers. His cognitive functions deteriorated rapidly and he died due to pneumonia in December, 2008, eleven months after the onset of symptoms.

\section{Discussion}

We presented the case of a 77 year-old patient with rapidly progressive dementia (RPD). Our initial diagnostic hypotheses included prion disease or autoimmune encephalopathy, but ancillary tests were not supportive. At this point, because of the rapid rate of cognitive decline, a brain biopsy was ordered which confirmed a diagnosis of cerebral amyloid angiopathy (CAA).

CAA is characterized by the deposition of amyloid proteins within leptomeningeal and cortical vessels, with the $\beta$-amyloid type being the most common ${ }^{3,4}$. CAA may manifest clinically not only as lobar hemorrhage - the most recognized manifestation - but also as ischemic infarctions, subarachnoid hemorrhage, transient focal neurological manifestations (caused by focal seizures or transient ischemic attacks) or cognitive decline ${ }^{3,5}$. 


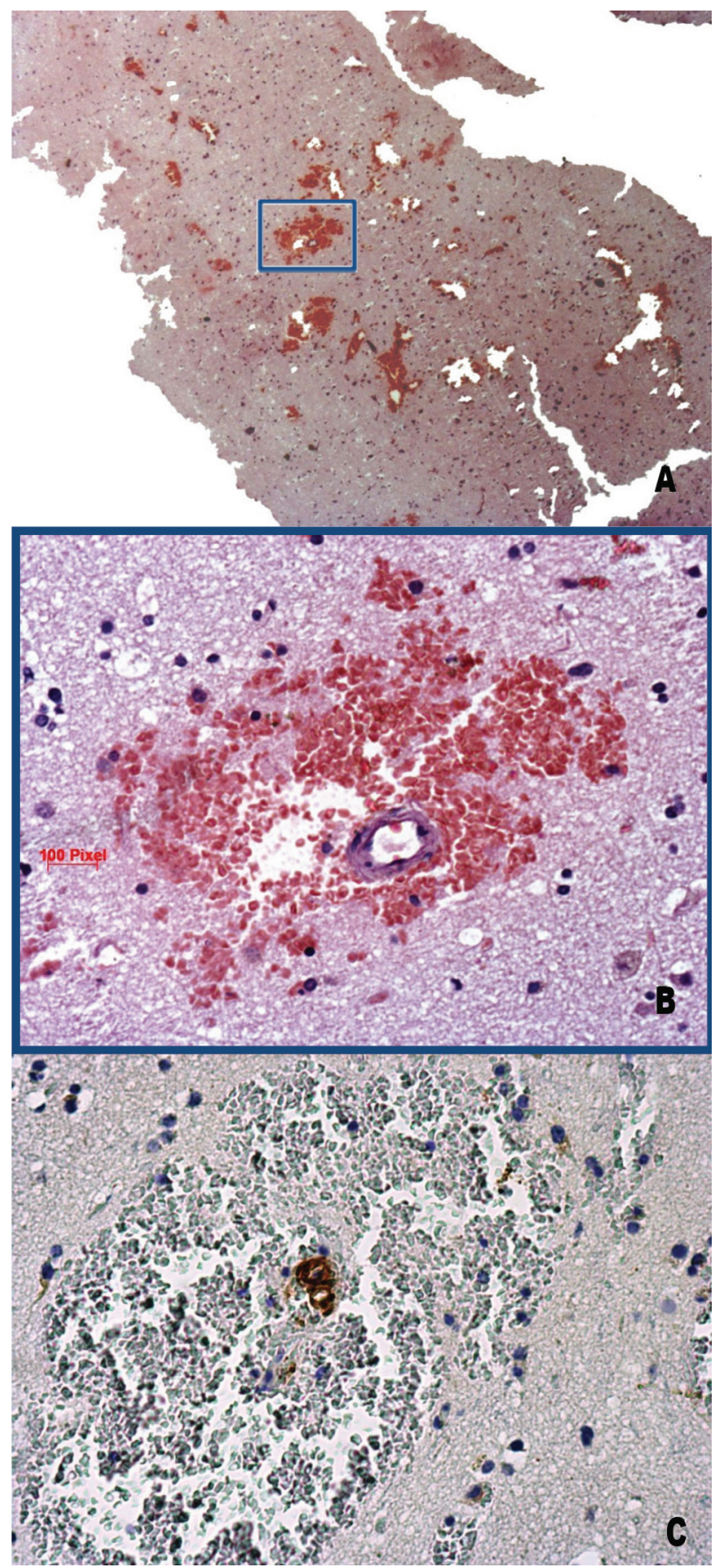

Figure 3. $[A]$ frontal cortex depicting multiple foci of micro-bleeding. HE. 25x. [B] Higher magnification of the boxed area in 3A. Note that the micro-bleeding is surrounding a vessel in which the wall is thickened by the deposition of amorphous material. 200x. [C] immunostaining against Amyloid- $\beta$. The brownish color of the vessel indicates positivity for Amyloid- $\beta$ protein. $400 x$

CAA has been linked to aging, dementia and Alzheimer's disease $(\mathrm{AD})$. It is known that the prevalence of CAA neuropathological findings increase with advancing age ${ }^{4,6}$.
CAA is also a recognized cause of cognitive impairment ${ }^{7}$, and was associated with a higher prevalence of dementia in a neuropathological study ${ }^{8}$.

MRI findings in CAA include cortico-subcortical microhemorrhages or petecchial hemorrhages (seen in gradient-echo sequences) $)^{9,10}$ and white matter hyperintensities . $^{4}$ Microbleeds on gradient-echo T2-weighted MRI have been associated with cognitive impairment, particularly executive impairment in nondemented individuals ${ }^{11,12}$. Although gradient-echo MRI is considered a sensitive test for CAA (by revealing hemorrhagic lesions $)^{10}$, in this case, a discrepancy between neuroimaging findings with a relative paucity of hypointense lesions in MRI T2*-weighted images and neuropathological findings of multiple microbleeds was noted. There is also the possibility that acute-appearance microbleeds may have occurred during the biopsy procedure which might explain the disparity.

White matter lesions in CAA are believed to be caused by chronic hypoperfusion of the deep white matter associated with vascular changes of long perforating arteries ${ }^{4}$. It is relevant, however, that despite the fact that white matter abnormalities are characteristic in CAA, severe and diffuse white matter hyperintensities (resembling subcortical arteriosclerotic encephalopathy) such as those seen in this case, are considered rare by some ${ }^{13}$.

CAA has also been associated with $\mathrm{AD}$, after the frequent observation of CAA in AD patients (between 78 and $\left.100 \%{ }^{14,15}\right)$, and because patients with $\mathrm{AD}$ and CAA have showed more widespread distribution than non-demented individuals with $\mathrm{CAA}^{6}$. Capillary occlusion or narrowing caused by CAA may explain the hypoperfusion that is believed to occur in the AD brain ${ }^{6}$ and thus may be a part of its pathophysiology ${ }^{16,17}$.

More recently, CAA and RPD have been linked with a form of inflammatory vasculopathy, known as cerebral amyloid inflammatory vasculopathy ${ }^{18}$ or amyloid angiopathy-related inflammation ${ }^{19,20}$. For reasons still unknown, a subset of patients with severe CAA may develop a picture of encephalopathy with subacute cognitive decline, seizures and/or headache that may respond (with both clinical and radiological improvement) to anti-inflammatory treatment ${ }^{19}$. Brain MRI reveals large and confluent areas of white matter hyperintensities in T2/FLAIR-weighted images and scattered microbleeds on gradient-echo sequences ${ }^{19}$. Neuropathological evaluation shows perivascular inflammation with mononuclear and multinucleated white blood cells surrounding vessels stained positive for $\mathrm{A} \beta$ (which raised the possibility of $A \beta$ being the trigger to immune response) as well as multiple microbleeds and small infarctions in the cerebral cortex besides rarefaction of the underlying white matter $^{20}$. Although the patient did not receive immunosup- 
pressive therapy, this form of inflammatory vasculopathy cannot be considered the diagnosis, as no inflammatory signs were found in the cerebral biopsy.

One may question whether CAA was the only cause of $\mathrm{RPD}$ in this patient. Indeed, one of the limitations of this report is the lack of sufficient neuropathological data to either confirm or exclude the diagnosis of AD. Only diffuse plaques were observed, and thus neither Braak \& Braak stage (based on the finding of neurofibrillary tangles) ${ }^{21}$ or Consortium to Establish a Registry for Alzheimer's Disease (CERAD) criteria (based on neuritic plaques) ${ }^{22}$ for AD were fulfilled. However, the brain fragment analyzed consisted of biopsy specimen and thus the co-morbidity of $\mathrm{AD}$ could not be ruled out. In contrast with CAA, which is more commonly and precociously found in the neocortex $\mathrm{x}^{6}$, neuritic plaques and neurofibrillary tangles are observed in the isocortex in more advanced phases of $\mathrm{AD}^{21}$ and a vascular amyloid finding is not directly correlated with amyloid plaque deposition ${ }^{23}$. The sensitivity of brain biopsy for the diagnosis of dementia should also be considered. As reviewed by Warren et al. ${ }^{24}$, across 17 studies, a specific diagnosis was achieved in $22-84 \%$ of brain biopsies. In a series of patients with rapidly progressive neurological conditions, Josephson et al..$^{25}$ found that brain biopsies had a sensitivity of $65 \%$ for the diagnosis (the most common being lymphoma and Creutzfeldt-Jakob disease). A positive ANA was found in low titers $(1: 80)$. However, this patient presented no other signs of systemic autoimmune disorder and a false positive ANA may be found in low serum dilutions in controls (with 1:160 being suggested as a cutoff) ${ }^{26}$.

On one hand, there are reports of CAA solely causing $\mathrm{RPD}^{5}$ while vascular dementia is known to be a cause of $\mathrm{RPD}^{1}$. On the other hand, CAA is known to lower the threshold for dementia caused by neurodegenerative conditions (such as AD) and RPD is usually associated in the context of CAA with amyloid angiopathy-related inflammation ${ }^{3}$ - which as stated above was not the case in this patient. The patient's clinical co-morbidities, such as anemia and poorly compensated diabetes (as shown by high glycosylated hemoglobin) could have also hastened the cognitive decline. Either way, irrespective of whether CAA was the sole cause of RPD in this patient or not, we believe this report serves to raise the awareness of CAA as a cause of RPD in patients with leukoencephalopathy and microhemorrhages.

\section{References}

1. Geschwind MD, Shu H, Haman A, Sejvar JJ, Miller BL. Rapidly progressive dementia. Ann Neurol 2008;64:97-108.

2. Folstein MF, Folstein SE, McHugh PR. "Mini-mental state”. A practical method for grading the cognitive state of patients for the clinician. J Psychiatr Res 1975;12:189-198.
3. Maia LF, Mackenzie IR, Feldman HH. Clinical phenotypes of Cerebral Amyloid Angiopathy. J Neurol Sci 2007;257:23-30.

4. Yamada M. Cerebral amyloid angiopathy: an overview. Neuropathology 2000;20:8-22.

5. Greenberg SM, Vonsattel JP, Stakes JW, Gruber M, Finklestein SP. The clinical spectrum of cerebral amyloid angiopathy: presentations without lobar hemorrhage. Neurology 1993;43:2073-2079.

6. Thal DR, Griffin WS, de Vos RA, Ghebremedhin E. Cerebral amyloid angiopathy and its relationship to Alzheimer's disease. Acta Neuropathol 2008;115:599-609.

7. Greenberg SM, Gurol ME, Rosand J, Smith EE. Amyloid angiopathy-related vascular cognitive impairment. Stroke 2004;35:2616-2619.

8. Pathological correlates of late-onset dementia in a multicentre, community-based population in England and Wales. Neuropathology Group of the Medical Research Council Cognitive Function and Ageing Study (MRC CFAS). Lancet 2001;357:169-175.

9. Walker DA, Broderick DF, Kotsenas AL, Rubino FA. Routine use of gradient-echo MRI to screen for cerebral amyloid angiopathy in elderly patients. AJR Am J Roentgenol 2004;182:1547-1550.

10. Knudsen KA, Rosand J, Karluk D, Greenberg SM. Clinical diagnosis of cerebral amyloid angiopathy: validation of the Boston criteria. Neurology 2001;56:537-539.

11. Werring DJ, Frazer DW, Coward LJ, et al. Cognitive dysfunction in patients with cerebral microbleeds on $\mathrm{T} 2{ }^{\star}$-weighted gradient-echo MRI. Brain 2004;127:2265-2275.

12. Yakushiji Y, Nishiyama M, Yakushiji S, et al. Brain microbleeds and global cognitive function in adults without neurological disorder. Stroke 2008;39:3323-3328.

13. Oide T, Takahashi H, Yutani C, Ishihara T, Ikeda S. Relationship between lobar intracerebral hemorrhage and leukoencephalopathy associated with cerebral amyloid angiopathy: clinicopathological study of 64 Japanese patients. Amyloid 2003;10:136-143.

14. Attems J, Lauda F, Jellinger KA. Unexpectedly low prevalence of intracerebral hemorrhages in sporadic cerebral amyloid angiopathy: an autopsy study. J Neurol 2008;255:70-76.

15. Tian J, Shi J, Mann DM. Cerebral amyloid angiopathy and dementia. Panminerva Med 2004;46:253-264.

16. Kumar-Singh S. Cerebral amyloid angiopathy: pathogenetic mechanisms and link to dense amyloid plaques. Genes Brain Behav 2008;7(Suppl 1):67-82.

17. Smith EE, Greenberg SM. Beta-amyloid, blood vessels, and brain function. Stroke 2009;40:2601-2606.

18. Harkness KA, Coles A, Pohl U, Xuereb JH, Baron JC, Lennox GG. Rapidly reversible dementia in cerebral amyloid inflammatory vasculopathy. Eur J Neurol 2004;11:59-62.

19. Kinnecom C, Lev MH, Wendell L, et al. Course of cere- 
bral amyloid angiopathy-related inflammation. Neurology 2007;68:1411-1416.

20. Eng JA, Frosch MP, Choi K, Rebeck GW, Greenberg SM. Clinical manifestations of cerebral amyloid angiopathy-related inflammation. Ann Neurol 2004;55:250-256.

21. Braak H, Braak E. Neuropathological stageing of Alzheimerrelated changes. Acta Neuropathol 1991;82:239-259.

22. Mirra SS, Heyman A, McKeel D, et al. The Consortium to Establish a Registry for Alzheimer's Disease (CERAD). Part II. Standardization of the neuropathologic assessment of Alzheimer's disease. Neurology 1991;41:479-486.

23. Lippa CF, Hamos JE, Smith TW, Pulaski-Salo D, Drachman
DA. Vascular amyloid deposition in Alzheimer's disease. Neither necessary nor sufficient for the local formation of plaques or tangles. Arch Neurol 1993;50:1088-1092.

24. Warren JD, Schott JM, Fox NC, et al. Brain biopsy in dementia. Brain 2005;128:2016-2025.

25. Josephson SA, Papanastassiou AM, Berger MS, et al. The diagnostic utility of brain biopsy procedures in patients with rapidly deteriorating neurological conditions or dementia. J Neurosurg 2007;106:72-75.

26. Tan EM, Feltkamp TE, Smolen JS, et al. Range of antinuclear antibodies in "healthy" individuals. Arthritis Rheum 1997;40:1601-1611. 